\title{
Postnatal Maturation of the Response of the Canine Sinus Node to Critically Timed, Brief Vagal Stimulation
}

\author{
ARTHUR S. PICKOFF, RODRIGO RIOS, ADRIENNE STOLFI, AND SHI NAN WANG \\ Section of Pediatric Cardiology, Department of Pediatrics, Tulane University School of Medicine, \\ New Orleans, Louisiana 70112
}

\begin{abstract}
We have previously demonstrated that vagal phase-response curves (PRC), which characterize the effects of critically timed, brief vagal stimuli on sinus node automaticity, exhibit a fundamentally different shape in the canine newborn than in the adult. In this study we analyzed the changes in sinus cycle length in response to critically timed, brief vagal stimuli, delivered to the decentralized cervical right and left vagosympathetic trunks, in two older age groups: 14 1-mo-old puppies (ages 21-36 d), and eight 2-mo-old puppies (ages 56-62 d). Vagal PRC were constructed by plotting the magnitude (percent change) of the vagal chronotropic response as a function of the phase of the cardiac cycle at which the vagus nerve was stimulated. At 1 mo of age adult-type PRC were observed, but in only six of the puppies (43\%) and only in response to right vagal stimulation. By 2 mo of age adulttype PRC were observed in seven of eight puppies $(88 \%$ ) in response to right vagal stimulation and in three of eight $(38 \%)$ in response to left vagal stimulation. Thus, clear developmental changes in the phase dependence of the vagal chronotropic response can be tracked over the first 2 mo of life in the dog. (Pediatr Res 35: 55-61, 1994)
\end{abstract}

Abbreviations

$A V$, atrioventricular

PRC, phase-response curve(s)

Previous in vivo and in vitro studies have demonstrated clearly that the effect of a brief vagal stimulus on heart rate or AV nodal conduction is highly dependent upon the phase within the cardiac cycle that the stimulus is applied $(1-12)$. Typically, this phase dependence is expressed as an absolute increase in the magnitude of the vagal response because a single, brief vagal stimulus is delivered progressively later within the cardiac cycle. The increase in the vagal response continues until a point is reached within the cardiac cycle that the stimulus is delivered sufficiently late that it is unable to affect the next beat. This defines the beginning of the latency period, or the interval from the earliest point in the cardiac cycle where the vagal stimulus elicits no response, to the onset of the next cardiac cycle (7). This phase dependence may have true physiologic significance because in vivo it is believed that vagal discharges do occur as short, discrete bursts of activity within the cardiac cycle (13). With the

Received April 1, 1993; accepted September 9, 1993.

Correspondence and reprints: Arthur S. Pickoff, M.D., Department of Pediatrics, Section of Cardiology, Tulane University School of Medicine, 1430 Tulane Avenue, New Orleans, LA 70112.

Supported by Grant HD 28929-01 from the National Institute of Child Health and Human Development to A.S.P. magnitude of the vagal response being partly dependent upon the phase of the cardiac cycle in which the burst occurs, a potential mechanism exists for fine, beat-by-beat regulation of heart rate and $\mathrm{AV}$ nodal conduction by the parasympathetic nervous system.

We have reported previously that classical phase dependence of the vagal response is not, however, observed in newborn animals (14). Specifically, we have shown that in the canine newborn phase-response curves generated in response to brief, critically timed vagal stimuli for both sinus cycle length and AV nodal conduction exhibit a different shape or configuration than reported in the adult. In the newborn, rather than a gradual increase in the magnitude of the vagal response as the stimulus is delivered progressively later within the cardiac cycle, we observed most typically no change in the magnitude of the response or, at times, even a decrease in the response. This suggested to us that the newborn might not have the same mechanism for fine regulation of heart rate and AV nodal conduction by the parasympathetic nervous system as the adult.

In this study we report the changes that occur during early postnatal development in the response of heart rate to brief, critically timed vagal stimulation. We demonstrate that in the dog there is a maturation of the response elicited to brief vagal stimulation during the first $60 \mathrm{~d}$ of life, with gradual emergence of the adult-type vagal phase-response curve. In addition, we provide evidence that the shape of the vagal PRC during this period of development is, in part, dependent upon the time course of acetylcholine release and hydrolysis.

\section{MATERIALS AND METHODS}

General preparation. The study was performed in two groups of young mongrel dogs. All experiments were approved by the Tulane University School of Medicine Advisory Committee for Animal Resources and were conducted in accordance with federal guidelines for the care and use of laboratory animals. The first group consisted of 14 puppies approximately $1 \mathrm{mo}$ of age (range, 21-36 d). The second group consisted of eight puppies approximately 2 mo of age (range, 56-62 d). The mean ( \pm SD) weight in the first group was $1449 \pm 308 \mathrm{~g}$ and $3249 \pm 733 \mathrm{~g}$ in the second group. Data (previously unreported) from two canine newborns, ages 6 and $17 \mathrm{~d}$ old are also presented. All were anesthetized with $30 \mathrm{mg} / \mathrm{kg}$ pentobarbital given intraperitoneally and placed on a mechanical ventilator (Harvard model 613 South Natick, MA). A cannula was placed in the left femoral vein for the administration of drugs. A second cannula was placed in the left femoral artery for monitoring arterial blood gases and blood pressure. Surface ECG lead II was also monitored. Via the right femoral vein a $4 \mathrm{~F}$ quadripolar electrode catheter was positioned within the high right atrium to record electrograms from this site and to provide a trigger for the delivery of appropriately timed vagal bursts (see below). Both 
cervical vagosympathetic trunks were isolated and divided, and the distal ends of each were prepared for electrical stimulation with a pair of platinum bipolar electrodes. The nerves were kept moistened with oxygenated, physiologic saline to prevent drying and to preserve nerve function. Propranolol (1 mg/kg) was administered i.v. $15 \mathrm{~min}$ before the beginning of data collection to eliminate sympathetic effects on sinus cycle length during the course of the experiments. In our model, this dose of propranolol completely abolishes the decrease in sinus cycle length caused by a test dose of isoproterenol $(0.20 \mu \mathrm{g} / \mathrm{kg}$ i.v. $)$. Surface ECG, blood pressure, and atrial electrograms were amplified and recorded at a paper speed of $100 \mathrm{~mm} / \mathrm{s}$ with a Gould TA-2000 chart recorder (Gould Inc., Cleveland, $\mathrm{OH}$ ). This permitted measurements of the change in sinus cycle length (measured as the AA interval in the right atrial electrode catheter) to the nearest $\pm 5 \mathrm{~ms}$.

Experimental design. Vagal stimuli were delivered at a constant current and with a pulse duration of $2 \mathrm{~ms}$ by a programmable stimulator (Bloom model DTU-210, Reading, PA). The strength of the applied stimulation current was chosen in an identical manner in both younger and older puppies. Vagal stimuli were delivered at a current intensity (in milliamperes) that produced a maximal increase in sinus cycle length without producing AV block when stimulating the nerve tonically at a frequency of $8 \mathrm{~Hz}$ for approximately $10 \mathrm{sec}$. In these experiments the mean current output used was $2.3 \pm 1.1 \mathrm{~mA}$ in the 1 -moold group and $3.4 \pm 1.1 \mathrm{~mA}$ in the 2 -mo-old group. In the experimental protocols, vagal stimuli were delivered as a single, brief train of five stimuli with an interpulse interval of $7 \mathrm{~ms}$. This single train was first delivered once, $10 \mathrm{msec}$ after the high right atrial electrogram was sensed (A-ST 10) and the effect on sinus cycle length (the AA interval recorded in the high right atrium electrode catheter) was determined. The single stimulus train was then delivered at other times within the cardiac cycle, scanning the entire cardiac cycle length randomly to include the following stimulation coupling intervals after the high right atrial electrogram was sensed (A-ST interval): 20, 40, 60, 80, 100, 125 , $150,175,200$... ms. One minute was allowed to pass in between each test train. In this way changes in sinus cycle length produced by a brief vagal train could be analyzed as a function of the timing of the vagal burst within the cardiac cycle. PRC were generated by plotting the percent change in sinus cycle length (percent change $=100[(\mathrm{~A}|\mathrm{~A} 2-\mathrm{A}| \mathrm{A} 1) / \mathrm{A} 1 \mathrm{~A} 1)]$, where $\mathrm{A} 1 \mathrm{~A} 1$ represents the control sinus cycle length and A1A2 the sinus cycle length following the brief vagal train, as a function of the A-ST interval. At the conclusion of each complete scan, the response to A-ST 10 was retested to be sure that the response at the end of the experimental protocol was the same as at the start of the experiment. The latency period of the phase-response curve was determined in each experiment and was defined as the interval from the earliest A-ST interval that prolonged the next sinus cycle by less than $5 \%$ to the beginning of that cardiac cycle.

In these experiments, we classified PRC as having either a neonatal-type ("N") configuration or an adult-type pattern ("A"). We have reported previously (14) that the most noticeable difference between neonatal and adult-type PRC is the shape of the PRC before the latency interval (adult-type PRC gradually increase in magnitude up until latency, whereas neonatal-type PRC remain flat or decrease). To classify PRC in this study, we determined the slope of each PRC through linear regression, from A-ST 10 through the maximum value of the PRC, and included all points up to the beginning of the latency interval (or until the magnitude of the PRC began to decrease by more than $10 \%$, indicating the approach to latency). We defined any PRC with an initial slope of $>+0.001$ as an adult-type PRC. Complete scans were obtained with right vagus nerve stimulation in all 22 puppies and with left vagus nerve stimulation in four of the 1mo-old puppies (omitted or abandoned in the others because of technical difficulties such as loss of nerve response), and in all eight of the 2-mo-old puppies.

In addition to phase response curves, we also plotted and analyzed vagal inhibition curves (curves that describe the effect of a brief vagal stimulus over time) as a tool for assessing the time dependent kinetics of the vagal response. The methods for constructing vagal inhibition curves have been well described previously (2). Briefly, at each A-ST coupling interval, the effect of the brief vagal train on sinus cycle length was measured for each subsequent cardiac cycle (A1A2, A2A3, A3A4. . A9A10) following the stimulus. The percent change in sinus cycle length was then plotted as a function of the expected ST-A interval (i.e. the interval from the brief vagal train to the expected time of discharge of each sinus beat). To characterize the kinetics of both onset and decay of the primary vagal response, the ascending and terminal limbs of the primary vagal response of the vagal inhibition curve were fitted by linear regression and the slopes of both limbs thus determined.

Statistical analysis. All statistical analyses were performed on data obtained during right vagal stimulation. Fisher's Exact test was used to compare the frequency of adult- and neonatal-type PRC in the two age groups. The maximum percent change in response to an $8 \mathrm{~Hz}$ vagal train was compared between the two age groups with a two-sample $t$ test. Multivariate analysis of variance, followed by univariate $F$ tests, was used to compare several variables of the neonatal- and adult-type PRC, as well as variables extrinsic to the PRC itself. The PRC variables included the percent change in response to the brief vagal train at A-ST 10 , the maximum percent change in response to a brief vagal train at any A-ST interval, the latency period, and the interval between the maximum percent change and the beginning of the latency period. Variables extrinsic to the PRC itself included the percent change in sinus cycle length in response to an $8 \mathrm{~Hz}$ vagal train $(8 \mathrm{~Hz}$ max \%), vagal stimulation current intensity (Stim $\mathrm{mA}$ ), the resting sinus cycle length (SCL), and the slopes of the ascending (A slope) and descending (D slope) limbs of the vagal inhibition curves. The Bonferroni correction for multiple comparisons was applied to all individual comparisons between the adult-type and neonatal-type PRC groups. Multiple regression analysis was used to assess the correlation with PRC shape of the variables extrinsic to the PRC itself. The general linear model used was phasics slope $=\mathrm{C}_{1}+\mathrm{C}_{2}(8 \mathrm{~Hz} \max \%)+\mathrm{C}_{3}(\mathrm{Stim} \mathrm{mA})$ $+\mathrm{C}_{4}(\mathrm{SCL})+\mathrm{C}_{5}$ (A slope) $+\mathrm{C}_{6}$ (D slope), with a constant $\left(\mathrm{C}_{1}\right)$ and five coefficients $\left(C_{2}-C_{6}\right)$. For all statistics, a $p$ value of $<0.05$ was used to indicate statistical significance. All values are expressed as means $\pm \mathrm{SD}$.

\section{RESULTS}

PRC configuration as a function of postnatal age. PRC generated in response to brief trains of vagal stimulation delivered to the right vagosympathetic trunk were classified as adult-type in six of the 141 -mo-old puppies (43\%) and neonatal-type in eight (57\%) (Fig. 1). In contrast, in the 2-mo-old puppies, an adulttype PRC was observed in seven of the eight puppies $(88 \%)$ and a neonatal-type PRC in only one (Fig. 2). The difference in the frequencies of the adult and neonatal PRC types between the two age groups was significant $(p<0.05)$.

Left vagal stimulation data were available in four of the 1-moold puppies and in all eight of the 2-mo-old puppies. Neonataltype PRC were seen in all four of the 1-mo-old puppies even though all were in puppies in which a clear adult-type PRC was observed in response to right vagal stimulation. In the 2-mo-old group, adult-type PRC curves were observed in only three of the eight puppies during left vagal stimulation; the remainder exhibited the neonatal pattern. Thus, a dissociation in the PRC configuration could be observed in the responses to brief right and left vagal stimulation. That is, an adult-type pattern could be observed in response to right vagal stimulation and a neonataltype pattern in response to left vagal stimulation.

Comparison of neonatal- and adult-type PRC. In comparing PRC classified as neonatal- versus adult-type, it was observed that the magnitude of the vagal chronotropic response was greater 

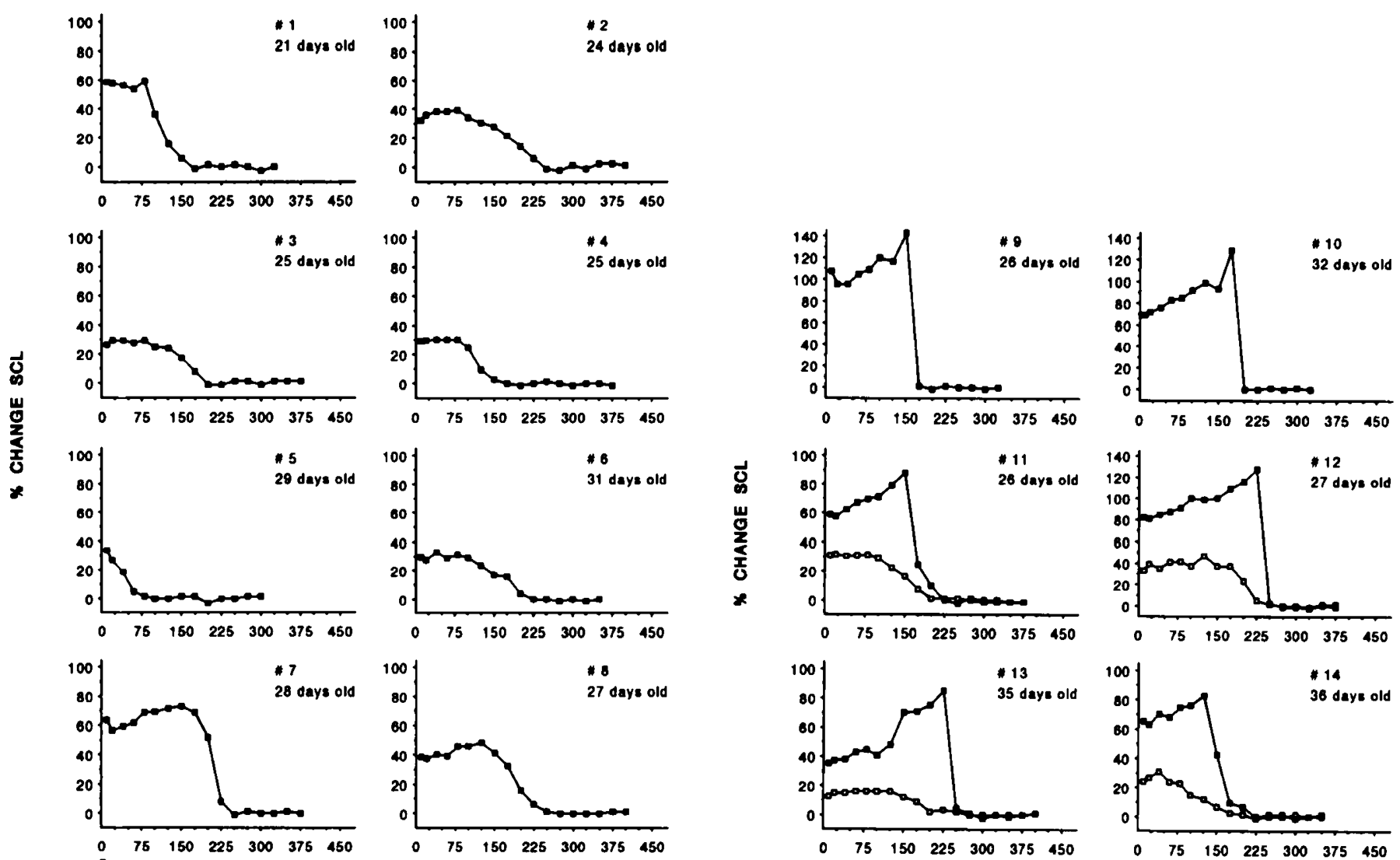

A

A-ST INTEAVAL (MSEC)

- RVS
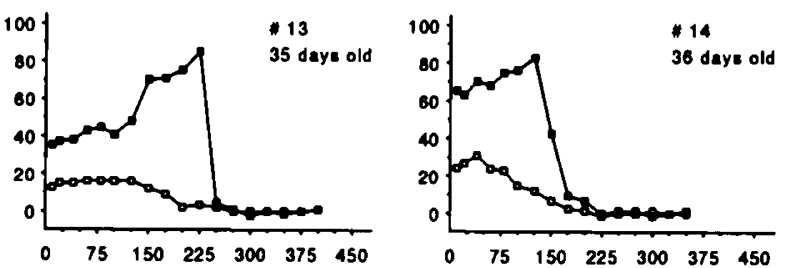

B

A-ST INTERVAL (MSEC)

- RVS OLVS

Fig. 1. Vagal PRC recorded in 14 puppies approximately 1 mo of age. PRC are illustrated in response to brief right vagal stimulation in all dogs, and both right and left vagal stimulation in puppy nos. 11-14. The PRC exhibit a typical neonatal shape ("N") in the first six examples $(A)$ and an adult shape ("A") in the last six $(B)$. The PRC of puppy nos. 7 and 8 are hybrids of both neonatal and adult types, with some increase in the vagal response noted as the A-ST interval is moved through the cardiac cycle, but with the overall change $<15 \%$. In the last four examples, vagal PRC in response to right vagal stimulation demonstrate a typical adult-type pattern, whereas PRC in response to left vagal stimulation are neonatal-type PRC.

in PRC that were classified as adult-type based on the slope of the initial portion of the PRC (Table 1). The slopes of the PRC classified as neonatal-type ranged from -0.0070 to 0.0008 (puppy nos. 1-8, 18), while those classified as adult-type ranged from 0.0016 to 0.0029 (puppy nos. 9-17, 19-22). Both the percent change in sinus cycle length in response to coupling interval A-ST 10 and the maximum percent change in sinus cycle length at any A-St interval were significantly greater in puppies with adult-type PRC. This suggests that the magnitude of the vagal chronotropic response may be linked to the shape of the vagal PRC. Although the vagal chronotropic responses were generally larger in the puppies with adult-type PRC, it should be noted that adult-type PRC could also be observed with relatively weak vagal chronotropic responses. As an example, in Figure 2, the PRC generated in response to left vagal stimulation in puppy no. 16 showed a clear adult-type pattern and yet the vagal chronotropic response ranged from only $36 \%$ at A-ST 10 to a maximum response of $61 \%$. Similarly, we have observed clear neonatal-type PRC in newborns with very large vagal responses. As an example of this, in Figure 3, a PRC is shown from a newborn dog (age $6 \mathrm{~d}$ ) in which the magnitude of the vagal response was very large ( $>90 \%$ change in sinus cycle length) and yet the shape of the PRC was clearly a neonatal type. These examples suggest that there may not be a definable threshold of magnitude of the vagal chronotropic response that uniquely determines the shape of the vagal PRC.

No significant difference was found in the latency periods of neonatal and adult-type PRC. There was, however, a significant difference noted in the interval separating the A-ST at which the maximum value of the PRC occurred and the onset of the latency period. In adult-type PRC, the interval separating the maximum PRC value from the onset of the latency period was significantly shorter than in neonatal-type PRC.

Relationship of response to tonic vagal stimulation, vagal stimulation intensity, and sinus cycle length to PRC shape. Consistent with previous developmental studies $(15,16)$ a general increase was observed in the magnitude of the change in sinus cycle length in response to a tonic $(8 \mathrm{~Hz})$ vagal stimulation train with increasing age. The maximum percent change in sinus cycle length during an $8 \mathrm{~Hz}$ tonic vagal train increased from $54 \pm 15 \%$ in the 1 -mo-old puppies to $73 \pm 19 \%$ in the 2 -mo-old puppies $(p<$ $0.05)$. The maximum percent change in sinus cycle length in response to tonic vagal stimulation was also greater in puppies with adult-type PRC compared with puppies with neonatal-type PRC (68 $\pm 11 \%$ versus $51 \pm 23 \%, p<0.01)$. However, in multiple regression analysis (Table 2 ), the magnitude of the response to tonic vagal stimulation was not significantly correlated with PRC shape. The intensity of the current used during vagal stimulation was not significantly different $(3.1 \pm 1.3 \mathrm{~mA}$ in puppies with adult-type PRC versus $2.1 \pm 0.8 \mathrm{~mA}$ in puppies with neonatal-type PRC, $p=0.06$ ), and was not correlated with PRC shape.

Because changes in sinus cycle length have been reported to alter the vagal PRC (7), the influence of sinus cycle length on PRC shape was evaluated. In those puppies with adult-type PRC, the resting sinus cycle length was $422 \pm 51 \mathrm{~ms}$ and in puppies 

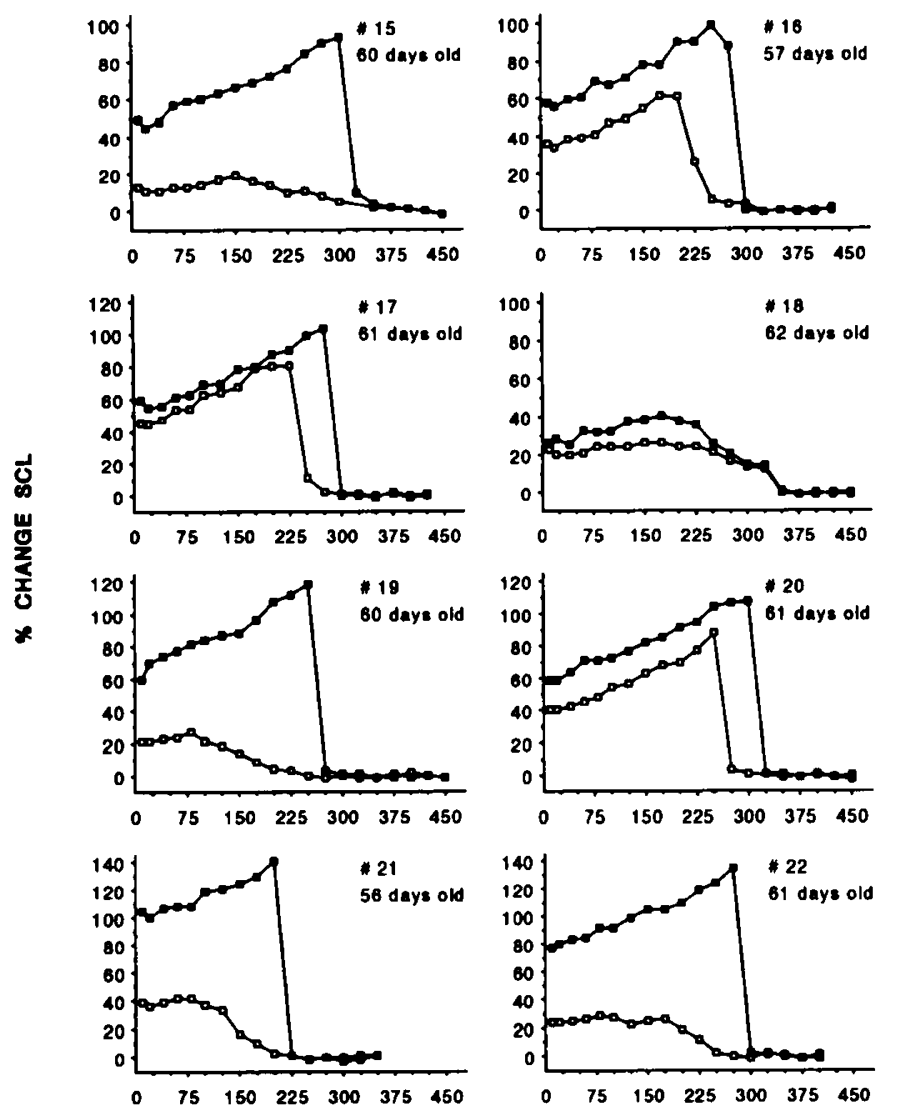

A-ST INTERVAL (MSEC)

- RVS O LVS

Fig. 2. Vagal PRC recorded in eight puppies approximately 2 mo of age. PRC for both right and left vagal stimulation are shown. In response to right vagal stimulation, the PRC configurations are adult-type ("A") in all except one puppy (no. 18). In response to left vagal stimulation, neonatal patterns ("N") are observed in five puppies and adult patterns are seen in three (nos. 16, 17, 20).

Table 1. Comparison of $P R C$ variables in adult-type vs neonatal-type $P R C^{*}$

\begin{tabular}{lrrc}
\hline \multicolumn{1}{c}{ Variable } & $\begin{array}{c}\text { Adult } \\
\text { response }\end{array}$ & $\begin{array}{c}\text { Neonatal } \\
\text { response }\end{array}$ & $p$ \\
\hline A-St 10 (\% change) & $68 \pm 21$ & $38 \pm 15$ & $<0.01$ \\
Maximum (\% change) & $112 \pm 22$ & $43 \pm 15$ & $<0.01$ \\
Latency period (ms) & $162 \pm 25$ & $179 \pm 38$ & NS $\dagger$ \\
Onset of latency (A-ST) - & $38 \pm 24$ & $139 \pm 36$ & $<0.01$ \\
$\quad$ maximum (A-ST) (ms) & & &
\end{tabular}

* All values represent the mean $\pm \mathrm{SD} . n=13$ for adult response; $n=$ 9 for neonatal response. Multivariate analysis of variance for all variables combined: $p<0.00001 ; p$ values in table are for individual comparisons between the two groups (univariate $F$ tests with Bonferroni correction for multiple comparisons).

† NS, no significant difference between the two groups.

with neonatal-type PRC it was slightly shorter $(389 \pm 51 \mathrm{~ms}, p$ $=0.15$ ). There was a great deal of overlap in the range of sinus cycle lengths in those with adult-type PRC (352-515 ms) and neonatal-type PRC (300-480 ms). In the multiple regression model, sinus cycle length was not correlated with PRC shape.

Vagal inhibition curves and PRC configuration. Because rapid kinetics for both the release and subsequent hydrolysis of acetylcholine are considered essential conditions for the critical phase dependence of the vagal chronotropic response as expressed in the adult-type PRC, vagal inhibition curves (curves that describe the effects of a brief vagal volley over a period of time) were

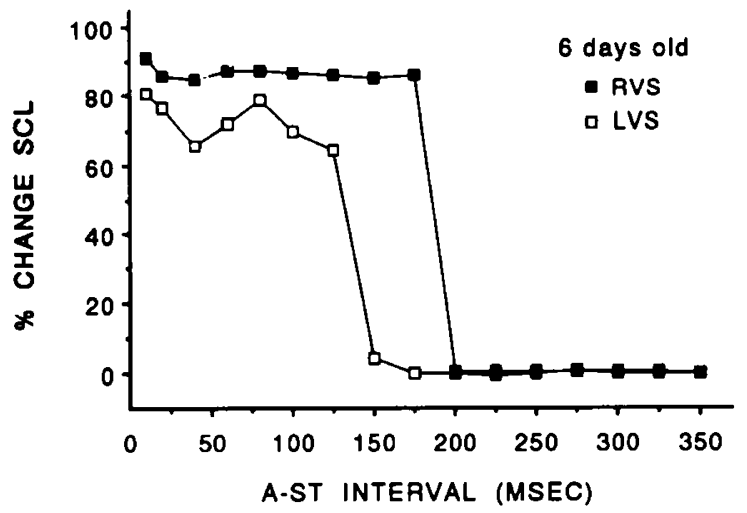

Fig. 3. Vagal PRC in response to right vagal stimulation in a very young, newborn $\operatorname{dog}(6 \mathrm{~d}$ old $)$. The magnitude of the vagal chronotropic response is quite large $(\sim 90 \%)$ yet the shape of the vagal PRC is clearly a neonatal-type (i.e. no change in the magnitude of the vagal response as the brief vagal train is delivered at progressively later A-ST intervals). The latency interval begins at approximately A-ST 200 and is very sharply defined.

Table 2. Multiple regression analysis of variables predicting PRC shape*

\begin{tabular}{lrrrrc}
\hline \multicolumn{1}{c}{ Variable $\dagger$} & $\begin{array}{r}\text { Regression } \\
\text { coefficient }\end{array}$ & SEE $\ddagger$ & \multicolumn{1}{c}{$t$} & $r$ & \multicolumn{1}{c}{$p$} \\
\hline Constant & -0.002 & 0.001 & -1.857 & & 0.083 \\
8 Hz max \% & 0.048 & 0.123 & 0.389 & 0.100 & 0.702 \\
Stim mA & 0.024 & 0.106 & 0.230 & 0.059 & 0.821 \\
SCL & 0.074 & 0.111 & 0.669 & 0.170 & 0.513 \\
A slope & 0.383 & 0.165 & 2.319 & 0.514 & 0.034 \\
D slope & -0.559 & 0.177 & -3.161 & -0.632 & 0.006 \\
\hline
\end{tabular}

* Overall regression multiple $r$ was $0.924, F=17.455, p<0.0001$.

† Eight $\mathrm{Hz}$ max \%, maximum percent change in response to an $8 \mathrm{~Hz}$ tonic vagal stimulation train; Stim $\mathrm{mA}$, vagal stimulation current intensity; SCL, resting sinus cycle length (ms); A slope, ascending slope of vagal inhibition curve; D slope, descending slope of vagal inhibition curve.

$\ddagger$ SEE, standard error of coefficient estimate.

constructed for each puppy. In the puppy [and as described by others (19)], these curves typically consisted of a large, primary wave of inhibition (thought to reflect the direct, hyperpolarizing effect of acetylcholine on the sinus pacemaker) followed by a longer, lower amplitude secondary wave of inhibition, lasting for approximately 3-5 s (Fig. 4). These two components were sometimes separated by a period of relative or absolute acceleration of the sinus rate in some puppies, bestowing a triphasic shape to the vagal inhibition curve.

Analysis of the ascending and descending limbs of the primary wave of inhibition demonstrated that differences in the kinetics of the development and decay of the primary vagal response were highly correlated with the vagal PRC shape (Table 2). Representative vagal inhibition curves from three puppies with neonatal-type PRC (puppy nos. 3, 4, 7) and adult-type PRC (puppy nos. 9, 11, 22) are shown in Figure 4. With respect to the ascending limb of the vagal inhibition curve, the adult-type PRC pattern was associated with a more rapid upslope compared with PRC with the neonatal-type pattern (adult, $37.66 \pm 16.03$; neonate, $3.34 \pm 2.14, p<0.01)$. In only one instance was there overlap of the values of the slope of the ascending limb in puppies with adult- and neonatal-type PRC. Similarly, the rate of decay of the vagal response, as reflected in the slope of the descending limb of the primary wave of vagal inhibition in the vagal inhibition curve, was also more rapid in puppies with adult-type PRC compared with those with neonatal-type PRC (adult, -1.84 \pm 0.28 ; neonate, $-0.94 \pm 0.31, p<0.01)$. No overlap was observed with respect to the slope of the descending limb in 
NEONATAL-TYPE PRC

ADULT-TYPE PRC
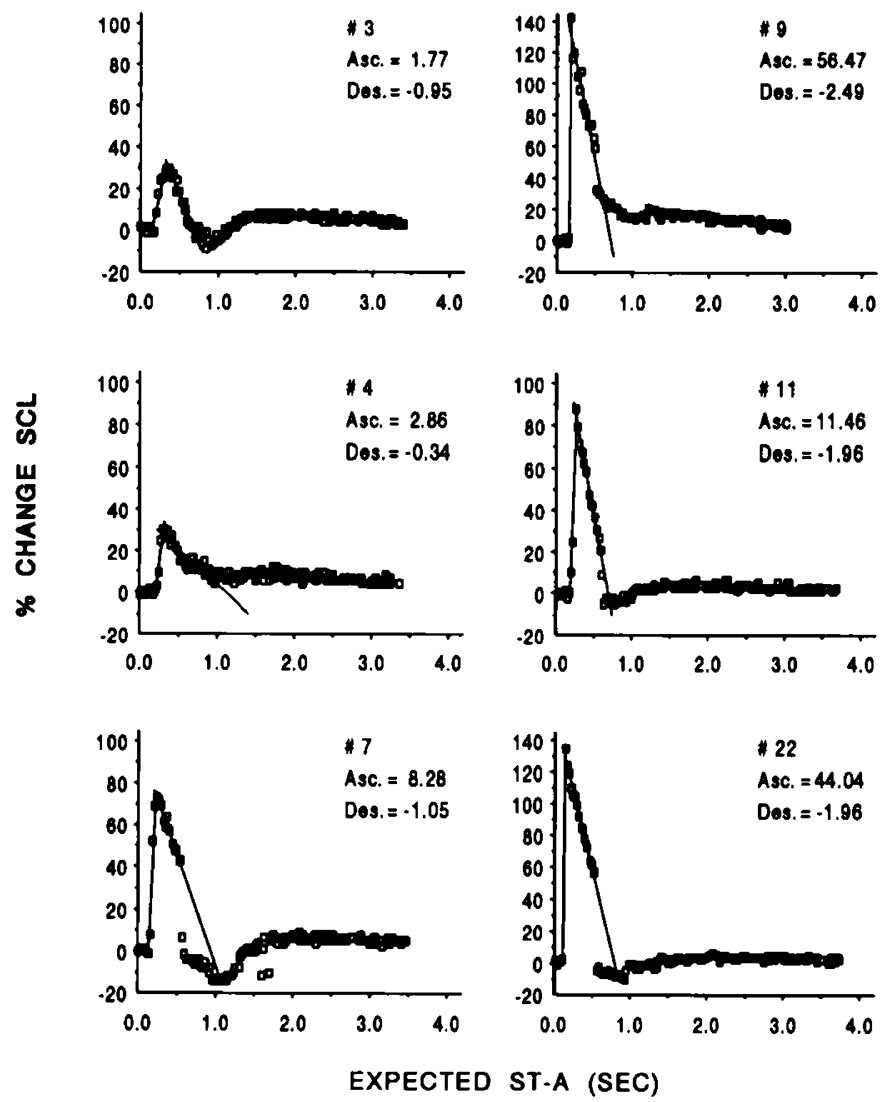

Fig. 4. Representative vagal inhibition curves in puppies with neonatal-type vagal PRC (left-hand column) and adult-type vagal PRC (righthand column). The ordinate represents the percent change in sinus cycle length and the abscissa the time from the vagal stimulus to the expected discharge of the sinus node (ST-A interval). The curves have a characteristic shape, with a primary wave of inhibition (first large peak) that lasts for $\sim 1 \mathrm{~s}$ and a secondary wave of inhibition that is of a lesser magnitude and lasts for at least $3 \mathrm{~s}$. As illustrated by puppy nos. 3, 7 and 22, these two waves of inhibition are often separated by a short period of relative or absolute acceleration of the sinus pacemaker rate. Both the ascending and descending limbs of the primary wave of inhibition were fit by linear regression (or, for ascending limbs with only two points, by connecting the peak response to the previous ST-A interval with no change in sinus cycle length) and the slopes were determined. Both ascending and descending slopes were significantly greater in those puppies with adulttype vagal PRC.

puppies with adult- versus neonatal-type PRC. Thus, with the emergence of the adult-type PRC configuration, vagal inhibition curves suggest that the kinetics of the vagal response become more rapid. That is, greater changes in the vagal chronotropic response (increases on the ascending limb, or decreases on the terminal limb of the vagal inhibition curve) occur in response to very small changes in the coupling interval (ST-A) of the vagal stimulus.

Inspection of neonatal- and adult-type PRC also supports differences in the kinetics of the vagal response. As described above, in puppies with adult-type PRC, a very narrow A-ST coupling range generally separates the maximum vagal response and the onset of the latency period ( $38 \pm 24 \mathrm{~ms})$. As examples, in the PRC of puppy nos. 9 and 10 (Fig. $1 B$ ), and nos. 15 and 17 (Fig. 2), there is only a 25-ms difference in the A-ST coupling intervals of the maximum vagal chronotropic response and the onset of latency. This would be possible only with a very discrete and rapid time course of the effect of a single vagal volley. In contrast, in many (but not all) neonatal-type PRC (as examples, puppy nos. 2, 3, 4, and 6), the A-ST intervals separating the maximum vagal response and the onset of latency can be quite large, approaching well over $100 \mathrm{~ms}(132 \pm 41 \mathrm{~ms})$. An extreme example of delayed vagal kinetics is illustrated in Figure 5. These PRC and vagal inhibition curves are from a 17-d-old puppy. A very gradual and prolonged decay of the vagal response begins at a very early phase ( A-ST 20 ) in the PRC (Fig. $5 A)$. The vagal inhibition curve (Fig. $5 B$ ) from the same puppy demonstrates a very prolonged slope of the terminal limb of the primary wave of inhibition (slope $=-0.228$ ). That the kinetics of development of the vagal chronotropic response is slow is also demonstrated by the PRC. Throughout the range of A-ST coupling intervals, the chronotropic response of the cardiac cycle in which the vagal train is delivered rapidly decays (A1A2), whereas the vagal chronotropic response is nearly maximal at every A-ST coupling interval in the following cardiac cycle (A2A3). In this example, vagal stimuli delivered no earlier than $260 \mathrm{~ms}$, and as long as $590 \mathrm{~ms}$ before the next cardiac cycle, prolong the cardiac cycle maximally. The slope of the ascending limb of the primary wave of inhibition of the vagal inhibition curve in this puppy was slow (1.954).

\section{DISCUSSION}

In this study we have tracked the maturation of the response of the sinus node to brief, critically timed vagal stimuli over the first $2 \mathrm{mo}$ of life in the young puppy. We have demonstrated
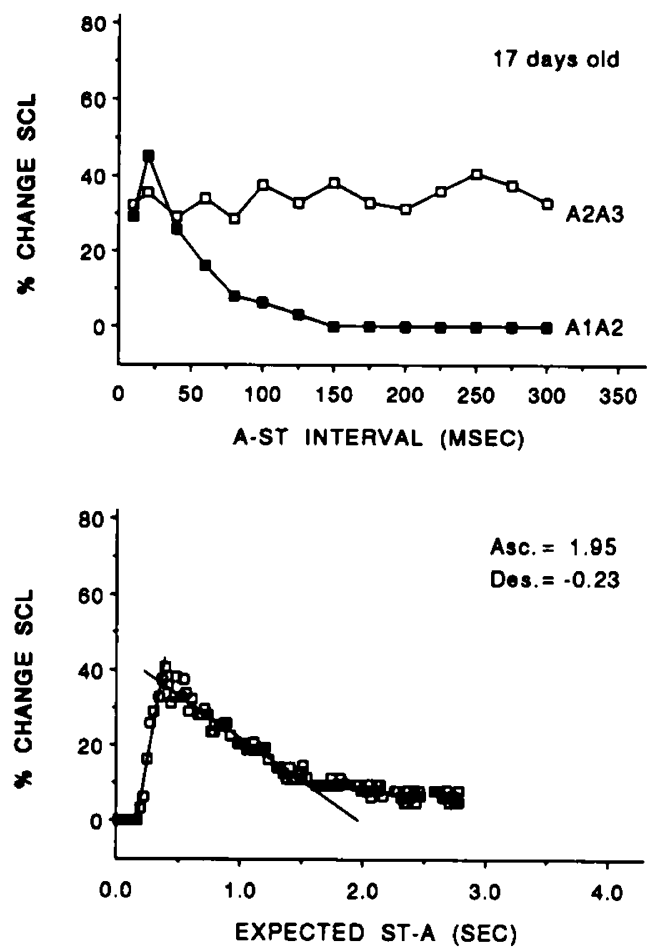

Fig. 5. Top, vagal $P R C$ in response to right vagal stimulation in a 17 . d-old puppy. Both the responses of the cardiac cycle in which the vagal train is delivered (AIA2) and the next cardiac cycle (A2A3) are shown. After a very early coupling interval (A-ST 20) where the PRC reaches its maximum value ( $\sim 5 \%$ change), there is a very gradual diminution in the vagal chronotropic response as the latency interval of the PRC (beginning at $\sim$ A-ST 125 ) is approached. The full chronotropic response is actually not realized until the next cardiac cycle (A2A3), where the cycle length is prolonged by $\sim 35 \%$ at each A-ST coupling interval. These features of the vagal PRC suggest very slow kinetics of the vagal response. Bottom, vagal inhibition curve of the same puppy. Note the very delayed terminal limb (and relatively slow upslope of the ascending limb) of the primary wave of inhibition. 
that over this period of time a significant change occurs in the phase dependence of vagal chronotropic responses. Specifically, we have shown that the vagal PRC undergoes a fundamental change in configuration: from the neonatal-type reported by our laboratory in canine neonates (14) to the classical adult-type PRC configuration reported in the adult. At 1 mo of age, vagal PRC generated in response to right vagal stimulation demonstrated the typical adult configuration in $43 \%$ of the puppies studied, compared with $<1 \%$ in the newborn period. This number increases to nearly $90 \%$ by 2 mo of life. Furthermore, by 2 mo of life, adult-type PRC configurations begin to emerge in response to left vagal stimulation. Thus, just as maturation of the vagal chronotropic response to tonic vagal stimulation has been tracked over the first months of life $(15,16)$, we have defined the early time course of maturation of yet another important physiologic aspect of parasympathetic function: critical time-dependence of the vagal response.

In addition to defining the time course of maturation of this particular aspect of parasympathetic function, our data also provide insight into possible factors that might contribute to the transformation of the vagal PRC from the neonatal-type to the adult-type in the first $60 \mathrm{~d}$ of life. The following variables have been reported to influence the shape and/or amplitude of the vagal PRC: 1 ) the resting sinus cycle length $(7), 2)$ the strength of the applied vagal stimulus $(1,4)$, and 3 ) the ambient acetylcholinesterase activity (17). No differences were noted in our study in the resting sinus cycle lengths of puppies with neonatalor adult-type vagal PRC. Furthermore, sinus cycle length was not correlated with PRC shape by multiple regression analysis. Similarly, the strength of the applied vagal stimulus in this study did not predict the shape of the PRC. We did note, however, that the absolute magnitude of the vagal chronotropic responses of the PRC were significantly greater in puppies with adult-type PRC compared with neonatal-type PRC. With a larger vagal response, not only does the magnitude of the vagal response increase but sinus node cell populations also tend to become more tightly synchronized (18). This is expressed in the vagal PRC as a very small difference in the A-ST interval that evokes the maximum vagal response and the onset of the latency period, as typically seen in the adult-type PRC.

Although a greater vagal chronotropic response was observed in puppies with an adult-type PRC shape, there are some critical observations that argue against this as being the single factor determining developmental differences in the shape of the vagal PRC. The first observation is that, in the puppy, very large vagal responses can, in fact, result in flat, neonatal-type vagal PRC (Fig. 3). The second observation relates to the phase difference between the maximum vagal response and the onset of the latency period. Although the phase difference is, in most instances, wider in neonatal-type PRC as compared with adulttype PRC, there are also very clear examples of neonatal-type PRC with very narrow phase differences between the maximum response and the onset of latency [Fig. 3; Fig. 1 in Yamasaki et al. (14)]. These examples would suggest that in these instances sinus nodal cells were, in fact, highly synchronized (i.e. the vagal stimulation intensity and/or response was maximal) and yet the shape of the vagal PRC was still not the classic adult-type PRC. Thus the magnitude of the vagal chronotropic response alone does not fully account for the different shapes of the neonataland adult type PRC.

Our analysis of vagal inhibition curves, as well as inspection of the vagal PRC, suggest to us that changes in the kinetics of the development of the vagal response and/or the decay of the vagal response may also contribute to the change in shape of the vagal PRC over the first 2 mo of life in the dog. Both the rates of development and decay of the primary wave of the vagal inhibition curve were significantly slower (with virtually no overlap of values) in puppies with neonatal-type PRC compared with those with adult-type PRC. The rate of decay of the primary vagal response has been considered a measure of the time course of action of acetylcholinesterase (19). That the neonatal PRC may, at least in part, be the result of an absolute or relative "insufficiency" of acetylcholinesterase in the immature canine heart has previously been proposed by us (14). This hypothesis is supported by the observations of Henning et al. (17), who demonstrated that acetylcholinesterase inhibition flattens the shape of the vagal PRC and increases the phase difference between the maximum vagal response and the onset of the latency period in adult dogs. A delayed time course of hydrolysis of acetylcholine and/or a delayed rate of development of the primary vagal response would both result in PRC in which the vagal response is less critically dependent on the phase within the cardiac cycle that the vagal stimulus is delivered. Alternatively, rather than an actual deficiency of acetylcholinesterase, the apparent delay in the time course of hydrolysis of acetylcholine may be simply a direct consequence of a greater dispersion over time of the release of acetylcholine (i.e. lack of synchronization). Direct measurements of acetylcholinesterase activity would be necessary to differentiate accurately between these possibilities. We do believe, however, that such kinetic considerations, in addition to the magnitude of the vagal chronotropic response, are the prime factors that determine the shape of the vagal PRC in these young puppies.

Finally, our experiments clearly demonstrate that the PRC generated in response to brief right and left vagal stimulation can be discordant. That is, it is possible to have a PRC in response to right vagal stimulation that is adult-type and, at the same time, a PRC in response to left vagal stimulation that is neonataltype. This provides very strong evidence that mechanisms that determine the shape of the vagal PRC do not involve the maturation of postsynaptic receptors, or intracellular signaling. It suggests that it is functional maturation of right and left parasympathetic neuronal elements proximal to the postganglionic synapse that is important in the transformation of the PRC from neonatal- to adult-type. That maturation of right and left vagal function could be functionally discordant is supported by the recent demonstration that there is minimal convergence of preganglionic vagal fibers on postganglionic neurons in the canine heart (20). Thus, a difference in the time course of maturation of the response to right versus left vagal nerve stimulation could be easily detectable in our canine model, given the absence of multiple interconnections between the two parasympathetic divisions.

In summary, these experiments track the maturation of the response of the canine sinus node to brief, critically timed vagal stimuli. Transformation of the response from the neonatal- to the adult-type PRC in response to right vagal stimulation is nearly complete by 2 mo of age. The response to left vagal stimulation appears to lag behind, but adult-type PRC begin to emerge at 2 mo of age. Our data suggest that the maturation of more than one aspect of parasympathetic function may be involved in the change in PRC configuration noted in early life. These factors relate to the magnitude of the vagal response and the kinetics of the primary wave of vagal inhibition. Maturation of this aspect of parasympathetic function has physiologic significance, as emergence of the adult-type PRC implies that the capability exists for very fine, beat-by-beat regulation of heart rate by the autonomic nervous system. This could conceivably be reflected as subtle differences in heart rate variability characteristics in the neonate compared to the adult. Such differences might prove to be of value in the characterization and subsequent detection of delayed maturation, or autonomic dysfunction in a variety of clinical settings.

\section{REFERENCES}

1. Dong Jr E, Reitz BA 1970 Effect of timing of vagal stimulation on heart rate in the dog Circ Res 27:635-646

2. Jalife J, Moe GK 1979 Phasic effects of vagal stimulation on pacemaker activity of the isolated sinus node of the young cat. Circ Res 45:595-607

3. Jalife J, Slenter VAJ, Salata JJ, Michaels DC 1983 Dynamic vagal control of pacemaker activity in the mammalian sinoatrial node. Circ Res 52:642-656 
4. Levy MN, Iano T, Zieske $H 1972$ Effects of repetitive bursts of vagal activity on heart rate. Circ Res 30:186-195

5. Levy MN, Martin PJ, lano T, Zieske H 1969 Paradoxical effect of vagus nerve stimulation on heart rate in dogs. Circ Res 25:303-314

6. Levy MN, Wexberg S, Eckel C, Zieske H 1978 The effect of changing interpulse intervals on the negative chronotropic response to repetitive bursts of vagal stimuli in the dog. Circ Res 43:570-576

7. Salata JJ, Gill RM, Gilmour Jr RF, Zipes DP 1986 Effects of sympathetic tone on vagally induced phasic changes in heart rate and atrioventricular node conduction in the anesthetized dog. Circ Res 58:584-594

8. Spear JF, Moore EN 1973 Influence of brief vagal and stellate nerve stimulation on pacemaker activity and conduction within the atrioventricular conduction system of the dog. Circ Res 32:27-41

9. Stuesse SL, Levy MN, Zieske H 1978 Phase-related sensitivity of the sinoatrial node to vagal stimuli in the isolated rat atrium. Circ Res 43:217-224

10. Wallick DW, Stuesse SL, Masuda Y 1986 Sympathetic and periodic vagal influences on antegrade and retrograde conduction through the canine atrioventricular node. Circulation 73:830-836

11. Warner MR, Loeb JM 1986 Phasic influences of vagal stimulation on atrioventricular conduction. Can J Physiol Pharmacol 66:1 198-1205

12. Yang T, Levy MN 1984 The phase-dependency of the cardiac chronotropic responses to vagal stimulation as a factor in sympathetic-vagal interactions. Circ Res 54:703-710

13. Katona PG, Poitras JW, Barnett GO, Terry BS 1970 Cardiac vagal efferent activity and heart period in the carotid sinus reflex. Am J Physiol 218:10301307

14. Yamasaki S, Stolfi A, Pickoff AS 1991 Characterization of responses of neonatal sinus and AV nodes to critically timed, brief vagal stimuli. Am J Physiol 260:H459-H464

15. Mace SE, Levy MN 1983 Neural control of heart rate: a comparison between puppies and adult animals. Pediatr Res 17:491-495

16. Pickoff AS, Stolfi A 1990 Modulation of electrophysiological properties of neonatal canine heart by tonic parasympathetic stimulation. Am J Physiol 258:H38-H44

17. Henning RJ, Masuda Y, Yang T, Levy MN 1987 Rate of acetylcholine hydrolysis affects the phase dependency of cardiac responses to vagal stimulation. Cardiovasc Res 21:169-176

18. Yang T, Jacobstein MD, Levy MN 1984 Synchronization of automatic cells in S-A node during vagal stimulation in dogs. Am J Physiol 246:585-591

19. Brown GL, Eccles JC 1934 The action of a single vagal volley on the rhythm of the heart beat. J Physiol (Lond) 82:211-241

20. Lang SA, Zieske H, Levy MN 1990 Insignificant bilateral convergence of preganglionic vagal fibers on postganglionic neurons to the canine heart. Circ Res 67:556-563 